
\title{
25 Research Suare \\ The Therapeutic Effect of Corneal Transplantation for Refractory Pseudomonas Aeruginosa Corneal Ulcer
}

\section{Yan Li ( $\sim$ swallow0310@126.com )}

Yantai Affiliated Hospital of Binzhou Medical University https://orcid.org/0000-0003-4786-5354

Ting Wang

Shandong Eye Hospital

Qiang Wang

Yantai Affiliated Hospital of Binzhou Medical University

Shanhao Jiang

Yantai Affiliated Hospital of Binzhou Medical University

\section{Xin Wang}

Moorfields Eye Hospital

Research article

Keywords: Pseudomonas aeruginosa corneal ulcer; LKP; Corneal transplantation

Posted Date: November 16th, 2019

DOl: https://doi.org/10.21203/rs.2.17414/v1

License: (c) (1) This work is licensed under a Creative Commons Attribution 4.0 International License. Read Full License 


\section{Abstract}

Purpose To observe the treatment outcome of corneal transplantation for advanced medically uncontrolled culture-proven pseudomonas aeruginosa corneal ulcer.Design Retrospective analysisSubjects and methods 26 patients (eyes) with refractory culture-positive pseudomonas aeruginosa corneal ulcer who failed to respond to drug therapy and underwent consecutive corneal transplant procedures in a hospital (2008.1-2018.8). Etiology, medical history, clinical features, surgical type, vision, recurrence, complications and treatment were recorded, and the relationship between postoperative recovery and selection of surgical method was analyzed.Results Of the 26 patients with pseudomonas aeruginosa corneal ulcer, 9 (34.6\%) received penetrating keratoplasty (PKP) and 17 $(65.4 \%)$ received lamellar keratoplasty (LKP). 22 patients (84.6\%) obtained a successful outcome through one corneal transplantation. Of the 9 patients who received PKP, 1 patient having graft rejection 6 months after surgery (endothelial type) obtained successful outcome through adequate drug treatment., while 1 case received success by graft repair combined with amniotic membrane transplantation on the 5 months postoperatively for fungal corneal graft ulcer. In the 17 patients underwent LKP, 2 received a second successful lamellar corneal transplantation for corneal graft melting 2 months after the first surgery. In all the 26 patients, the corneal infection was effectively brought under control by corneal transplantation, and none of them had recurrent ulcers during at least 6 months' follow-up. The visual acuity was significantly improved at the last follow-up compared with that before surgery. The postoperative visual acuity of patients underwent LKP was better than that of those who underwent PKP ( $p=0.018$. Conclusions Corneal transplantation can effectively treat refractory pseudomonas aeruginosa corneal ulcer worsening despite adequate medical treatment and improve eyesight. Compared with PKP, LKP can be the main surgical method to treat refractory pseudomonas aeruginosa corneal ulcer.

\section{Introduction}

Bacterial corneal ulcer, as a kind of microbial keratitis, is still the main cause of eye diseases and blindness in the world, especially in developing countries[1-6]. Among gram negative bacteria, pseudomonas aeruginosa is the most common[7]. It has been reported that pseudomonas aeruginosa is the most important pathogen in refractory bacterial corneal ulcer[8]. Pseudomonas aeruginosa corneal ulcer, a serious blinding eye disease, with rapid onset and development, whose treatment is very difficult, can lead to corneal perforation, endophthalmitis, acute vision loss even the prolapsus of eye viscera. Therefore, timely and effective treatment is very necessary. Current treatments include drug therapy, amnion transplantation, conjunctival flap occlusion, penetrating keratoplasty, and lamellar keratoplasty. A large number of studies have reported the treatment of pseudomonas aeruginosa corneal ulcer with drug therapy, amniotic membrane transplantation, conjunctival flap covering and so on. However, few studies have reported corneal transplantation for the treatment of pseudomonas aeruginosa corneal ulcer since 1964[8-11]. Corneal transplantation can not only completely remove bacteria, but also improve the transparency of the cornea and significantly increase visual acuity[12-15]. This study discussed the 
clinical characteristics, surgical indications, surgical effects and complications of refractory pseudomonas aeruginosa corneal ulcer treated with corneal transplantation in the hospital.

\section{Subjects And Methods}

This retrospective case analysis was approved by the Ethics Committee of the institute and complied with the Helsinki Declaration. We retrospectively analyzed the medical records of patients diagnosed with refractory pseudomonas aeruginosa corneal ulcer and received corneal transplantation in the hospital from January 2008 to August 2018. We analyzed the demographics of these patients, ulcer characteristics, results of smear and culture tests, and antibiotic sensitivity, methods of corneal transplantation, and postoperative recovery. The effects were evaluated by infection control, visual acuity recovery, and complications.

Pseudomonas aeruginosa corneal ulcer was diagnosed using the following methods: (1) clinical slit-lamp and anterior segment optical coherence tomography (AS-OCT) (OPTOVUE) examination revealed the modality and depth of the corneal ulcer respectively, (2) corneal scrapings at admission showed a positive pseudomonas aeruginosa culture result, (3) confocal microscopy examination (HRT3-RCM) imaged no fungal hyphae or acanthamoeba cysts to exclude fungal and amoeba infection respectively. Of all the 26 patients (eyes), 6 eyes (23.1\%) with corneal perforation and 2 eyes $(7.7 \%)$ with imminent corneal perforation were not given confocal microscopy examination. Each patient underwent B-scan ultrasounds in order to exclude endophthalmitis. Definition of refractory pseudomonas aeruginosa corneal ulcer: (1) drug treatment is ineffective; (2) the ulcer is larger than $6 \mathrm{~mm}$ in diameter, or deeper than $1 / 2$ corneal thickness, or smaller but located within $3 \mathrm{~mm}$ of the optic axis $\mathbb{(}(3)$ corneal perforation or imminent corneal perforation.

According to the operating regulations of our hospital, all patients were given corneal scrapings, bacterial culture and drug sensitivity test before taking any antibiotics. Specific methods: according to standard cultivation of microorganism separation procedures[16,17], ophthalmologist scraped both the base and edge of the ulcer with sterile blades under aseptic conditions after instillation of $4 \%$ Xylocaine, then inoculated the samples in blood and chocolate culture media, which also subjected Gram's stain and $10 \%$ potassium hydroxide wet microscopy, and meanwhile swabbed them to broth for enriched culture with sterile swabs to identify bacteria and fungi species and test antibiotic susceptibility.

Drug treatment: all patients received two or three broad spectrum antibiotic eye drops such as levofloxacin eye drops, $10 \%$ ceftazidine eye drops $囚$ tobramycin eye drops (Alcon, Fort Worth, TX, USA) once every half an hour, along with $1 \mathrm{~g}$ ceftazidine intravenous drip 3 times a day, $0.5 \mathrm{~g}$ levofloxacin intravenous drip once a day, and ofloxacin eye ointment (Santen, Osaka, Japan) at night. Then adjust the medication according to the drug sensitivity result. Surgical treatment should be considered if timely and adequate medication fails or the ulcer worsens for two weeks.

Penetrating corneal transplantation for those with Descemet membrane invasion or even perforation, otherwise with lamellar corneal transplantation. Penetrating corneal transplantation procedure: with 
manual dissection technique, the abnormal corneal tissue was cut out by a trephine with a diameter 0.25 $\mathrm{mm}$ larger than the ulcer and the DX-preserved donor corneal graft was sutured into the graft bed with $10 / 0$ nylon thread. Lamellar keratoplasty procedure: the necrotic corneal tissue was continuously exfoliated layer by layer until the left portion was clear. The glycerine-preserved donor graft was fixed onto the implant bed with 10/0 nylon thread by intermittent suture.

Postoperative treatment: Three to five days after surgery, all eyes were treated with tobradex eye ointment 4 times daily, subsequently with $0.1 \%$ or $0.02 \%$ fluorometholone eye drops 4 times a day according to the control of inflammation 5-7 days after surgery, which was decreased gradually within two weeks. $1 \%$ Cyclosporin A eye drops 3 times daily and tobradex eye ointment once each night were given when the epithelium repaired. 1\% Cyclosporin A eye drops and $0.02 \%$ fluorometholone eye drops were given 2 times a day, besides with tobradex eye ointment once per week three months after surgery.

Statistical analysis: SPSS 17.0 (SPSS, Inc, Chicago, Illinois, USA) was used for data analysis. A value of $p<0.05$ was considered statistically significant.

\section{Results}

Demographic data: this study included 20 males (76.9\%) and 6 females $(23.1 \%)$, with an average age of $46.8 \pm 16.8$ years (13-71 years). 16 right eyes $(61.5 \%)$ and 12 left eyes (38.5\%) were respectively affected. These patients included 18 farmers (69.2\%), 3 workers (11.5\%), 3 students $(11.5 \%)$ and 2 retirees $(7.7 \%)$. Males and farmers are more common, and most of them are 41-60 years old (Figure 1).

Clinical features: among the 26 patients, $14(53.9 \%)$ had a history of corneal trauma, $3(11.5 \%)$ had a history of wearing a contact lens, and $9(34.6 \%)$ had no obvious cause. Of the 26 patients, $22(84.6 \%)$ had anterior chamber hypopyon. Most patients had no systemic disease (76.9\%). Hypertension (7.7\%) was the most common associated systemic conditions, and myopia was the most common associated ocular disease (6\%). At admission, 4 patients $(15.4 \%)$ had high intraocular pressure $(>21 \mathrm{mmHg}$ measured by a Tono-Pen tonometer), 3 patients (11.5\%) had meibomian gland dysfunction and glaucoma, 1 patient (3.8\%) had lagophthalmus and glaucoma, and 1 patient (3.8\%) had Fuchs corneal endothelial dystrophy (Table 1). The average course of disease was $6.3 \pm 6.5$ days (1囚30 days) before patients came to our hospital. The average size of corneal ulcer was $8.5 \pm 2.2 \mathrm{~mm}(3 \otimes 12 \mathrm{~mm})$, and the average diameter of corneal graft was $9.1 \pm 2.1 \mathrm{~mm}(3.75 \otimes 12.25 \mathrm{~mm})$. For the smallest ulcer with a diameter of $3 \mathrm{~mm}$, the infiltration reached two thirds of corneal thickness, while the largest ulcer, $12 \mathrm{~mm}$ in diameter, invaded the corneoscleral limbus.

Results of culture and drug sensitivity test of corneal scraping: there were a large number of pus cells in each corneal spatula in 26 patients, and gram negative bacilli was detected in 14 patients, with a positive rate of 53.8\%. Pseudomonas aeruginosa was grown in culture in all 26 patients. Drug susceptibility test results: pseudomonas aeruginosa cultured from corneal smear of 26 patients, 24 cases were sensitive to tobramycin, 24 sensitive to ceftazidime, 23 sensitive to levofloxacin, and for the three kinds of drugs, 19 cases were sensitive to all of them, 6 cases were sensitive to two kinds of them, and 1 case was only 
sensitive to cephalosporin. Among all pseudomonas aeruginosa of the 26 patients, 15 were resistant to ceftriaxone, whose minimum inhibitory concentration (MIC) was more than 32 , and no resistance was found in 3 patients.

Postoperative recovery: In all the 26 patients, the corneal infection was effectively brought under control by corneal transplantation (Figure 2, Figure 3), and none of them had recurrent ulcers during at least 6 months' follow-up. For all the 26 patients, 22 patients (84.6\%) obtained successful outcome through one corneal transplantation. 9 patients received penetrating keratoplasty and 17 received lamellar keratoplasty. Of the 9 patients who received PKP, 1 patient having graft rejection 6 months after surgery (endothelial type) obtained successful outcome through adequate drug treatment, while 1 case received success by graft repair combined with amniotic membrane transplantation on the 5 months postoperatively for fungal corneal graft ulcer. In the 17 patients underwent LKP, 2 received a second successful lamellar corneal transplantation for corneal graft melting 2 months after the first surgery. The best corrected visual acuity of all patients at the last follow-up was significantly improved compared with that before surgery (Table 2). The postoperative visual acuity of patients underwent lamellar keratoplasty was better than that of those who underwent penetrating keratoplasty (Figure 4).

\section{Discussion}

The pseudomonas aeruginosa, as a kind of conditional pathogenic bacteria and cause of hospital acquired infection, can induce multiple organ damage, such as respiratory tract, urinary system, nervous system, eyes, ears, nose, throat and so on, and even sepsis heavier and illness, treatment more difficult. In the eye, pseudomonas aeruginosa may cause keratitis, corneal perforation, sharp vision loss and even endophthalmitis[18], which may be related to its virulence[19]. Therefore, timely and effective treatment is particularly important. Current treatments include drug therapy, amnion transplantation, conjunctival flap occlusion, penetrating keratoplasty, and lamellar keratoplasty. It has been reported that although frequent drugs can control inflammation to a certain extent, they are toxic to corneal epithelium, and vision was affected for corneal scarring[20]. Both amniotic membrane transplantation and conjunctival flap occlusion promoted epithelial healing, but the bacteria could not be completely removed, and neither visual acuity was improved[21-25]. Corneal transplantation, thoroughly removing bacteria, could improve the transparency of the cornea and significantly increased vision. Compared with the penetrating corneal transplantation, lamellar corneal transplantation has the advantages of more donors, smaller rejection, less complications, and a more ideal visual effect[26-28]. In this study, of the 26 patients, the corneal infection was effectively controlled by corneal transplantation. Twenty-two patients (84.6\%) achieved therapeutic success after one corneal transplantation, consistent with the results of the relevant studies[29]. Of the 9 patients underwent PKP, 1 patient had graft rejection 6 months after surgery, while 1 case underwent fungal corneal graft ulcer on the 5 months postoperatively. In the 17 patients underwent LKP, 2 had corneal graft melting 2 months after the first surgery. The best corrected visual acuity of all patients was significantly improved at the last follow-up compared with that before surgery, and lamellar keratoplasty was more conducive to the improvement of postoperative visual acuity than penetrating keratoplasty. 
It has been reported that bacterial corneal ulcer was mostly caused by corneal trauma\ocular surface disease and wearing corneal contact lens [30,31]. Our study showed that, the most common cause of pseudomonas aeruginosa corneal ulcer was corneal trauma, which may be a result of the occupations of our patients, as most of them were agricultural or other out-doors workers.

As the gold standard for the diagnosis of bacterial corneal ulcer, corneal spatula and bacterial culture are indispensable. In our study, there were a large number of pus cells in each corneal sample in 26 patients, and gram negative bacilli was detected in 14 patients, with a positive rate of $53.8 \%$, which was consistent with related reports[32,33]. Drug susceptibility test results: pseudomonas aeruginosa cultured from corneal smear of 26 patients, 24 cases were sensitive to tobramycin, 24 sensitive to ceftazidime, 23 sensitive to levofloxacin, and for the three kinds of drugs, 19 cases were sensitive to all of them, 6 cases were sensitive to two kinds of them, and 1 case was only sensitive to cephalosporin. The result was consistent with relevant reports[32,34]. Among all pseudomonas aeruginosa of the 26 patients, 15 were resistant to ceftriaxone, whose minimum inhibitory concentration (MIC) was more than 32 , and no resistance was found in 3 patients.

\section{Conclusions}

In conclusion, corneal transplantation can effectively treat refractory pseudomonas aeruginosa corneal ulcer and improve eyesight. Lamellar keratoplasty has the advantages of more donors, smaller rejection, less complications, and a more ideal visual effect, and can be the main surgical method to treat refractory pseudocycosis corneal ulcer. The deficiency of this study is that the number of cases is so small that analysis of related factors for corneal transplantation in the treatment of refractory pseudomonas aeruginosa corneal ulcer was not statistically significant, and further clinical data should be collected in the future to better guide future clinical work.

\section{Abbreviations}

PKP: penetrating keratoplasty; LKP: lamellar keratoplasty; AS-OCT: anterior segment optical coherence tomography; MIC: minimum inhibitory concentration.

\section{Declarations}

\section{Acknowledgements}

Thanks all the ophthalmologists participated in the research.

\section{Ethics approval and consent to participate}

The study was adhered to the tenets of the Declaration of Helsinki. Written informed consents were obtained from the patient.

\section{Consent for publication}


Not applicable.

\section{Availability of data}

All data generated or analyzed during the research are contained in the manuscript

\section{Competing interests}

None

\section{Funding}

None

\section{Authors' contributions}

Design of the study: YL and TW. Sample collection: YL and XW. Result analysis and manuscript preparation: YL and SJ. Revision of manuscript: QW and TW. All authors read and approved the final manuscript.

\section{Author details}

${ }^{1}$ Shandong University, Jinan 250012, China

${ }^{2}$ Department of Ophthalmology, Yantai Affiliated Hospital of Binzhou Medical University, Yantai 264100, Shandong Province, China

${ }^{3}$ Shandong Eye Hospital, Shandong Eye Institute, Shandong Academy of Medical Sciences, Jinan 250021, Shandong Province, China

\section{References}

1. Tabbara KF, Ross-Degnan D. Blindness in Saudi Arabia. JAMA. 1986 Jun 27;255(24):3378-3384.

2. Herretes $S$, Wang $X$, Reyes JM. Topical corticosteroids as adjunctive therapy for bacterial keratitis. Cochrane Database Syst Rev. 2014 Oct 16;(10):CD005430. doi: 10.1002/14651858.CD005430.pub3.

3. McLeod SD, LaBree LD, Tayyanipour R, et al. The importance of initial management in the treatment of refractory infectious corneal ulcers. Ophthalmology. 1995 Dec;102(12):1943-1948.

4. Whitcher JP, Srinivasan M, Upadhyay MP. Corneal blindness: a global perspective. Bulletin of the World Health Organization, 2001;79(3):214-221

5. Rachwalik D, Pleyer U. Bakterielle Keratitis. Klin Monbl Augenheilkd. 2015 Jun; 232(6): 738-744. doi: 10.1055/s-0035-1545994. Epub 2015 Jun 17

6. Robaei D, Watson S. Corneal blindness: a global problem. Clin Exp Ophthalmol. 2014 Apr;42(3):2134. doi: 10.1111/ceo.12330. No abstract available 
7. Rautaraya B, Sharma S, Ali MH, et al. A 31/2-Year Study of Bacterial Keratitis From Odisha, India. Asia Pac J Ophthalmol 2014;3(3): 146-150

8. Ti SE, Scott JA, Janardhanan P, et al. Therapeutic keratoplasty for advanced suppurative keratitis. Am J Ophthalmol. 2007;143(5):755-762

9. Malik SR, Singh G. Therapeutic Keratoplasty in Pseudomonas pyocyaneus corneal ulcers. Br J Ophthalmol. 1971 May;55(5):326-30. No abstract available

10. Gudkova LS, Berdoiants SA. Keratoplasty in the overall treatment of Pseudomonas aeruginosa infection of the cornea. Oftalmol Zh. 1980;35(7):445-6

11. Ricci A. Corneal Transplantation in Corneal Ulcer Caused By Pseudomonas Aeruginosa. Ophthalmologica. 1964;147:259-63

12. Gain P, Jullienne R, He Z, et al.Global Survey of Corneal Transplantation and Eye Banking. JAMA Ophthalmol. 2016 Feb;134(2):167-73

13. Tan DT, Dart JK, Holland EJ, et al. Corneal transplantation. Lancet. 2012 May 5;379:1749-61

14. Chow SP, Hopkinson CL, Tole DM, et al. Stability of visual outcome between 2 and 5 years of following corneal transplantation in the UK. National Health Service Blood and Ocular Tissue Advisory Group and Contributing Ophthalmologists (OTAG Study 23).Br J Ophthalmol. 2018 Jan;102(1):37-41

15. Yalniz-Akkaya Z, Burcu A, Doğan E,et al. Therapeutic penetrating keratoplasty for infectious and noninfectious corneal ulcers. Int Ophthalmol. 2015 Apr;35(2):193-200

16. J.G. Collee, R.S. Miles, Tests for isolation of bacteria, 13th ed., in: J.G. Collee, J.P. Duguid, A.G. Fraser, et al. (Eds.), Mackie and MacCartney Practical Medical Microbiolog, vol. 2, Churchill Livingstone, New York, 1989, pp. 456-481

17. Gopinathan U, Garg P, Sharma S, et al. Review of epidemiological features microbiological diagnosis and treatment outcome of microbial keratitis: experience over a decade. Indian J Ophthalmol. 2009;57:273Y279

18. Mena KD, Gerba CP. Risk Assessment of Pseudomonas aeruginosa in Water. Rev Environ Contam Toxicol. 2009;201:71-115

19. Dhakhwa K, Sharma MK, Bajimaya S, et al. Causative organisms in microbial keratitis, their sensitivity pattern and treatment outcome in western Nepal. Nepal J Ophthalmol 2012; 4: 119-27

20. Austin A, Lietman T, Rose-Nussbaumer J. Update on the Management of Infectious Keratitis. Ophthalmology. 2017 Nov;124(11):1678-1689

21. Dakhil TAB, Stone DU, Gritz DC. Adjunctive Therapies for Bacterial Keratitis. Middle East Afr J Ophthalmol. 2017 Jan-Mar;24(1):11-17

22. Saini JS, Sharma A, Grewal SP. Chronic corneal perforations. Ophthalmic Surg 1992;23:399-402.

23. Khodadoust A, Quinter AP. Microsurgical approach to the conjunctival flap. Arch Ophthalmol (Chicago, III: 1960) 2003;121:1189-93. 
24. Chen JH, Ma DH, Tsai RJ. Amniotic membrane transplantation for pseudomonal keratitis with impending perforation. Chang Gung Med J 2002;25:144-52

25. Malhotra C, Jain AK. Human amniotic membrane transplantation:

Different modalities of its use in ophthalmology. World J Transplant 2014;4:111-21

26. Tian L, Song XS, Xie LX. Research of the change tendency of corneal transplantation indications from 2000 to 2009. Zhonghua Yan Ke Za Zhi. 2011 Jul;47(7):623-7

27. Xie L, Shi W, Liu Z,etal. Lamellar keratoplasty for the treatment of fungal keratitis. Cornea. 2002 Jan;21(1):33-7

28. Geerling G, Duncker GI, Krumeich J,et al. Lamellar keratoplasty.Back to the future?! Ophthalmologe. 2005 Dec;102(12):1140-8, 1150-1

29. Ti SE, Scott JA, Janardhanan P, et al. Therapeutic keratoplasty for advanced suppurative keratitis. Ophthalmol.2007 143(5):755-762

30. Green M, Apel A, Stapleton F. Risk factors and causative organisms in microbial keratitis. Cornea. 2008;27:22-27.

31. Ibrahim YW, Boase DL, Cree IA. Epidemiological characteristics, predisposing factors and microbiological profiles of infectious corneal ulcers: the Portsmouth corneal ulcer study. $\mathrm{Br} \mathrm{J}$ Ophthalmol. 2009;93:1319-1324

32. Hongyok T, Leelaprute W. Corneal Ulcer Leading to Evisceration or Enucleation in a Tertiary Eye Care Center in Thailand: Clinical and Microbiological Characteristics. J Med Assoc Thai. 2016 Feb;99 Suppl 2:S116-22

33. Chan TC, Li EY, Wong VW, et al. Orthokeratology-associated infectious keratitis in a tertiary care eye hospital in Hong Kong. Am J Ophthalmol. 2014 Dec;158(6):1130-1135.e2. doi:

10.1016/j.ajo.2014.08.026. Epub 2014 Aug 23

34. Gupta S,Mittal S,Nayak N,et al. In Vitro Antibiotic Susceptibility of Pseudomonas aeruginosa Corneal Ulcer Isolates. Ocul Immunol Inflamm.2015 23(3):

\section{Tables}

Table 1 Systemic and ocular diseases $(n=26$ patients) 


\begin{tabular}{llllll}
\hline Systemic diseases & No. & $\%$ & ocular diseases & No. & \% \\
\hline None & 20 & 76.9 & None & 9 & 34.6 \\
Hypertension & 2 & 7.7 & Myopia & 6 & 23.1 \\
Diabetes & 1 & 3.8 & Glaucoma & 4 & 15.4 \\
Hypertension and & 1 & 3.8 & Meibomain gland & 3 & 11.5 \\
Diabetes & & & dysfunction & & \\
& & & and Glaucoma & & \\
Epilepsy & 1 & 3.8 & Meibomain gland & 2 & 7.7 \\
& & & dysfunction & & \\
Hepatitis B & & 3.8 & Lagophthalmus & 1 & 3.8 \\
& & & and Glaucoma & & \\
& & & Fuchs corneal endothelial & 1 & 3.8 \\
Total & & & dystrophy & & \\
\hline
\end{tabular}

Table 2: Final BCVA vs Preoperative UCVA 


\begin{tabular}{|c|c|c|}
\hline & Preoperative & At the Last Follow-up \\
\hline Light perception & $7(26.9)$ & $0(0)$ \\
\hline Hand motions & $14(53.8)$ & $0(0)$ \\
\hline Counting fingers & $4(15.4)$ & $0(0)$ \\
\hline$>$ Counting fingers & $1(3.8)$ & $6(23.1)$ \\
\hline \multicolumn{3}{|l|}{$\leq 20 / 200$} \\
\hline$>20 / 200$ & $0(0)$ & $8(30.8)$ \\
\hline \multicolumn{3}{|l|}{$\leq 20 / 60$} \\
\hline$>20 / 60$ & $0(0)$ & $7(26.9)$ \\
\hline \multicolumn{3}{|l|}{$\leq 20 / 40$} \\
\hline$>20 / 40$ & $0(0)$ & $5(19.2)$ \\
\hline \multicolumn{3}{|l|}{$\leq 20 / 25$} \\
\hline Total & 26 & 26 \\
\hline \multicolumn{3}{|c|}{ Values are represented as no. eyes (\%). } \\
\hline \multicolumn{3}{|c|}{ BCVA: Best-corrected Visual Acuity } \\
\hline \multicolumn{3}{|c|}{ UCVA: Uncorrected Visual Acuity } \\
\hline
\end{tabular}

\section{Figures}

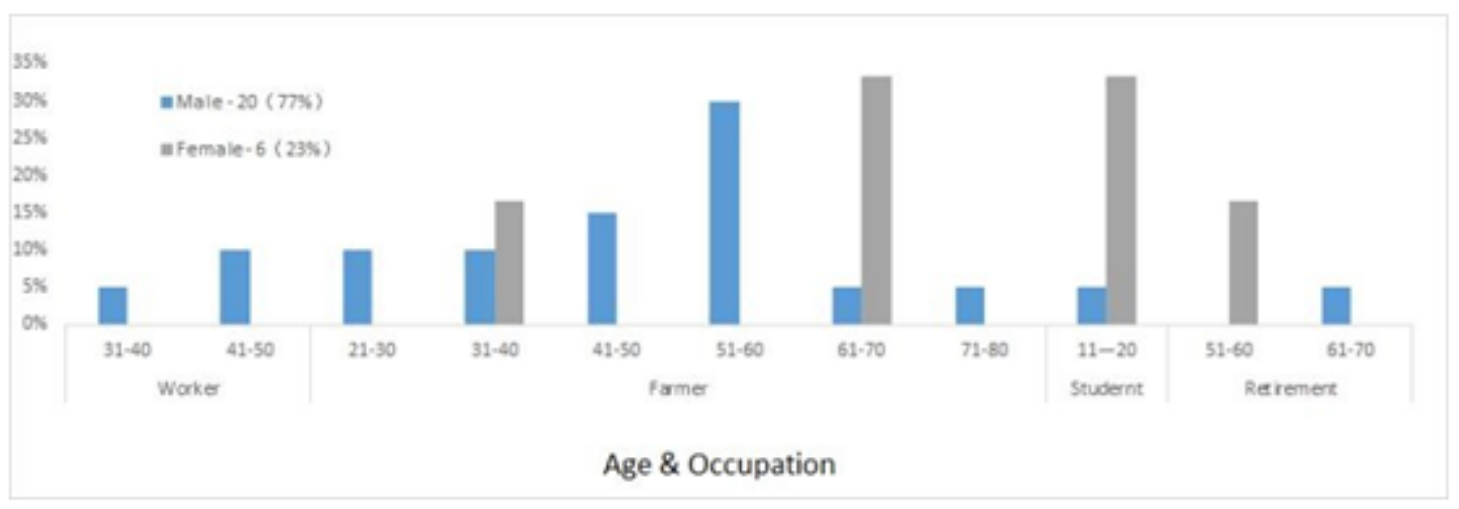

Figure 1

Demographic data of 26 patients with pseudomonas aeruginosa corneal ulcer

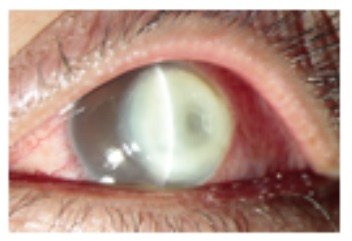

(a)

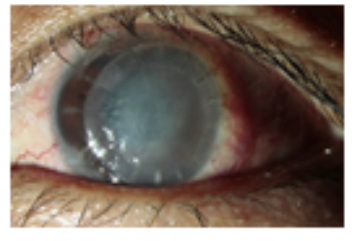

(b)

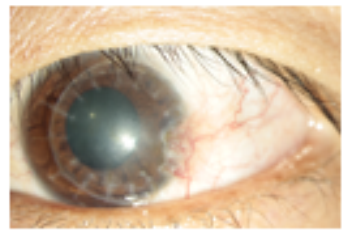

(c)

Figure 2 
LKP for pseudomonas aeruginosa corneal ulcer

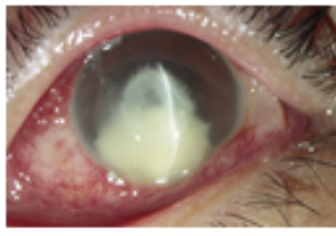

(a)

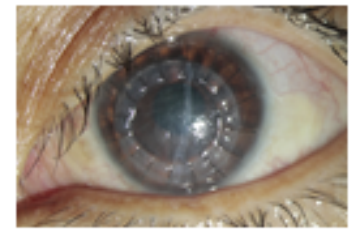

(b)

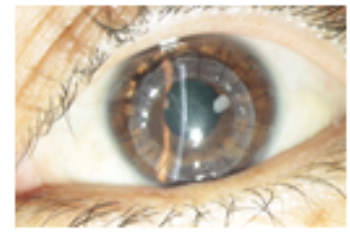

(c)

\section{Figure 3}

PKP for pseudomonas aeruginosa corneal ulcer

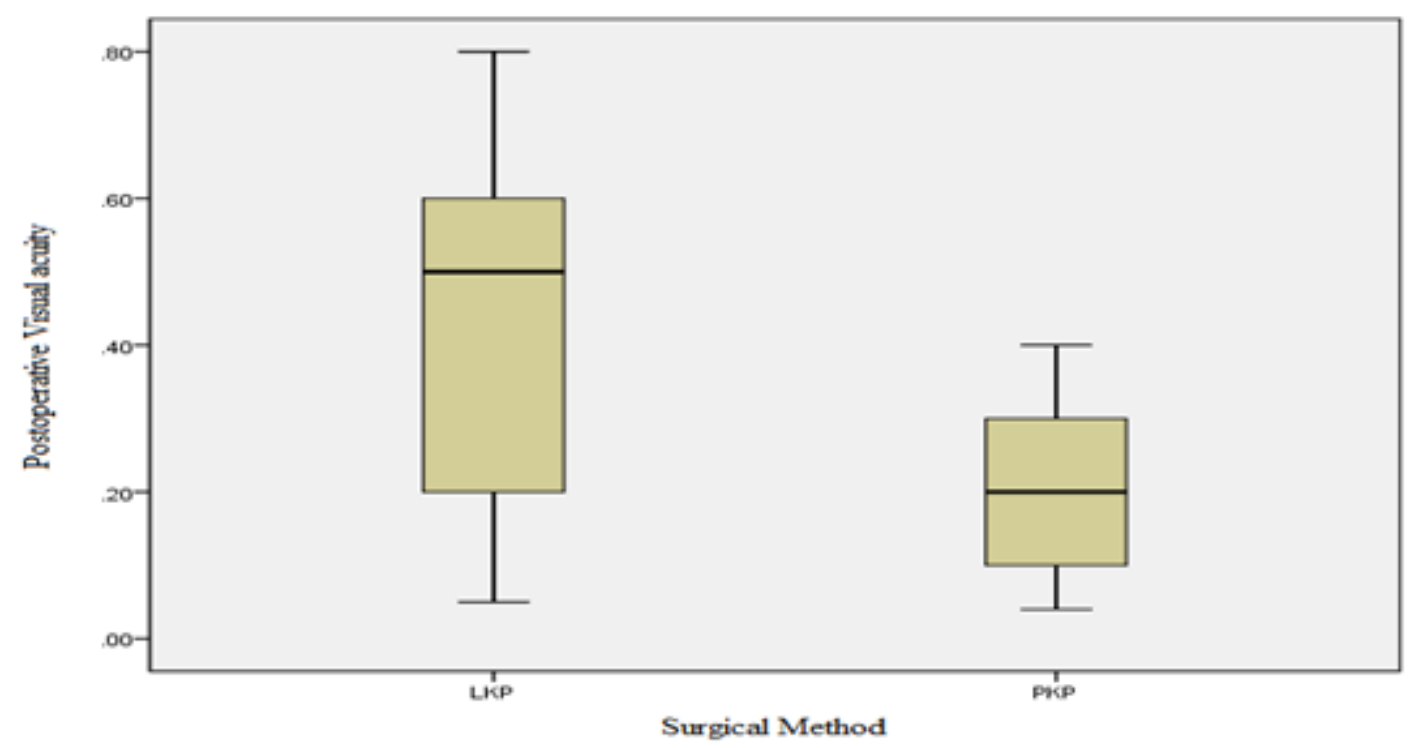

Figure 4

Postoperative visual acuity for patients underwent LKP and PKP 